\title{
Pediatric Inflammatory Neck Mass
}

\author{
Christian J. Hochstim, MD, PhD ${ }^{1}$ \\ Anna H. Messner, $M D^{2, *}$
}

\author{
Address \\ ${ }^{1}$ Clinical Otolaryngology, Department of Otolaryngology-Head and Neck Surgery, \\ Keck School of Medicine of USC, 4650 Sunset Blvd, Los Angeles, CA, 90027, USA \\ ${ }^{*}, 2$ Otolaryngology/Head \& Neck Surgery, Department of Otolaryngology-Head \& \\ Neck Surgery, Stanford University School of Medicine, 801 Welch Rd., Stanford, \\ CA, 94305, USA \\ Email: annamessner@stanford.edu
}

Published online: 21 July 2016

(C) Springer International Publishing AG 2016

This article is part of the Topical Collection on Otolaryngology

Keywords Neck mass · Cervical lymphadenitis · Neck abscess

\section{Opinion statement}

The treatment of pediatric inflammatory neck masses depends upon the underlying etiology and may include both medical and surgical interventions. The most common causes of bacterial cervical lymphadenitis include Staphylococcus aureus (increasingly methicillin-resistant S. aureus), and Streptococcus pyogenes. A contrast-enhanced computed tomography (CT) scan can provide an accurate means of diagnosing whether an abscess is present which might need surgical drainage. Recent studies, however, have focused on the ability to diagnose abscesses using imaging studies that limit the patient exposure to radiation, including the use of ultrasound, magnetic resonance imaging (MRI) and low-voltage CT scanning. Recent database studies have demonstrated trends in the incidence and management of deep neck abscesses from 2000 to 2009. The incidence of retropharyngeal abscesses has increased while the incidence of peritonsillar abscesses, parapharyngeal abscesses or combined space deep neck infections has remained the same. Rates of incision and drainage for retropharyngeal abscesses have decreased, while for peritonsillar abscesses rates of incision and drainage have increased with a corresponding decrease in tonsillectomy rates. Fusobacterial infections have been associated with peritonsillar abscesses and deep neck space infections in association with Lemierre syndrome. These infections are treated with culture-directed antibiotics and surgical drainage when indicated and the prognosis is generally good when identified early. Kawasaki disease (KD) is a potential non-infectious/idiopathic cause of inflammatory pediatric neck mass. In addition to cervical lymphadenopathy, patients with Kawasaki disease may present with retropharyngeal cellulitits or abscess. It is critical that the patient with KD be identified within 10 days of illness onset so the child can be treated with intravenous immunoglobulin which has unequivocally been shown to decrease the incidence of potentially life-threatening cardiac aneurysms. 


\section{Introduction}

Neck masses are commonly encountered in pediatric otolaryngology. The causes are varied and may include inflammatory, neoplastic, vascular or congenital causes. This chapter focuses on the inflammatory causes of pediatric neck masses. The history should include a detailed account of the duration for which the mass has been present, whether it has been growing, and if so, how rapidly. Any associated symptoms such as fever, pain, neck stiffness, trismus, or recent upper respiratory tract infection should be identified. The history should also include information of any travel history, sick contacts or potential exposure to different pathogens. On physical examination the clinician should assess whether the mass is tender, its location, size, any associated overlying erythema or skin changes and any evidence of fluctuance or drainage. Patients' physical exam findings should be followed to assess for response to therapy.

Imaging studies can be helpful in determining the diagnosis and appropriate treatment, but are not always necessary. They are particularly helpful in determining whether or not an abscess is present. Recent studies have examined alternatives to traditional contrast-enhanced computed tomography (CT) scanning in an effort to reduce the amount of radiation exposure to the patient. Alternatives to traditional CT include the use of ultrasound, MRI or low voltage CT scanning [1-4]. Treatment for pediatric inflammatory neck masses depends on the underlying etiology and options include medical management, surgery, and supportive care.

\section{Treatment}

\section{Use of imaging in the diagnosis of abscess}

Contrast-enhanced CT scan is a well-established and accurate means of diagnosing the presence of an abscess in cases of deep neck space infections. Despite its effectiveness, there have been increasing concerns recently about the potential detrimental effects of radiation exposure as a result of CT scans, especially in children. It is estimated that for every 600,000 children in the USA who received head or abdominal CT scans, 500 will die of cancer which is directly related to the CT [5]. The risk of brain cancer may triple in children under 15 as a result of the radiation exposure of 2-3 head CT scans $[6 \bullet \bullet]$. As a result of these serious concerns, there have been recent studies examining alternatives to CT scanning in the diagnosis of neck abscesses.

\section{Low-voltage CT scanning}

Contrast enhanced CT scans can demonstrate the presence of an abscess as a central hypodense mass surrounded by a hyperdense rim. Reduced tube voltage of $80 \mathrm{kVp}$ compared to the standard $120 \mathrm{kVp}$ results in a significant reduction in radiation exposure. Reduced-voltage CT was found to be equally effective to standard CT in diagnosing peritonsillar abscesses [7]. In addition CT scans with reduced voltage results in increased iodine contrast attenuation of soft tissue structures providing superior image contrast. [2•] The lower voltage CT images of peritonsillar abscesses were found to have improved sharpness and demonstration of 
inflamed soft tissue and also improved delineation of cervical vessels. The findings that reduced voltage CT can provide equal diagnostic accuracy with more clear images in addition to lower radiation exposure should lead to further investigation and strong consideration of more broad clinical application of this method.

\section{Ultrasound}

A retrospective study compared the accuracy of CT scan $(n=101)$ versus ultrasound $(n=39)$ in the diagnosis of pediatric lateral neck abscesses against the gold standard of incision and drainage. Ultrasound compared favorably with much higher specificity than CT (100 vs. $18 \%$ ) but lower sensitivity than CT (53 vs. $68 \%$ ) [3•]. Ultrasound can also be used intraoperatively to assist in localization of an abscess and to confirm successful abscess drainage. Recently, a case was reported using intraoperative transcervical ultrasound to assist with transoral drainage of a parapharyngeal abscess [1]. In addition to avoiding radiation exposure, ultrasound offers potential advantages over CT by avoiding the need for sedation in the pediatric patient, and the scan is less expensive than CT. Diagnostic accuracy, however, may be limited depending on the operator.

\section{Magnetic resonance imaging (MRI)}

MRI is a sensitive imaging modality for identifying inflammatory changes in soft tissue and can be useful in pediatric infections. MRI has been used to examine acute mastoiditis in adult and pediatric patients and it was observed that total opacification of the tympanic cavity and the mastoid, intense intramastoid enhancement, perimastoid dural enhancement, bone erosion, and extracranial complications are more frequent in children [4]. Given concerns over radiation exposure with CT scanning, MRI represents a potential alternative with the advantage of fine soft tissue detail. The disadvantages of MRI include a longer scanning time often requiring sedation/anesthesia in the pediatric patient; lack of availability in many emergency departments, and increased cost.

\section{Bacteriology of head and neck infections}

\section{Staphylococcus aureus}

S. aureus, including both methicillin-sensitive (MSSA) and methicillinresistant strains (MRSA), is an important cause of pediatric head and neck infections with high prevalence. Recent studies suggest that $S$. aureus is the most common organism isolated from pediatric neck abscesses, followed by Streptococcus pyogenes [8, 9]. A recent study from North Carolina of 76 children showed that $93 \%$ of culture-positive infections were caused by $S$. aureus in infants ( $<12$ months) compared to $59 \%$ in children between 13 months and 5 years of age. Of the 
S. aureus isolates, $51 \%$ were MRSA and $49 \%$ were MSSA. African American infants were found to have a 3-fold risk for methicillin resis-

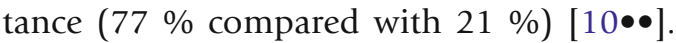

A review study from Pennsylvania of 178 patients aged 2 months to 12 years treated with parapharyngeal or retropharyngeal space infections showed that 60/178 (34\%) required surgical drainage and the remainder were treated with intravenous antibiotics only. Cultures obtained from those who were treated surgically showed that MRSA was found more

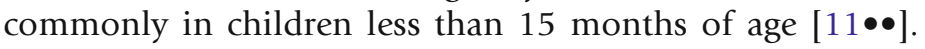

\section{Bacteriology of neck abscesses in the neonatal period}

Deep neck abscesses are rare in the neonatal period (birth to 1 month). Most infants who develop a neck abscess do not have any risk factors for a deep neck abscess. The most common clinical presentation of a deep neck abscess in the newborn is neck swelling, feeding and respiratory problems. Group B streptococcus, S. aureus, and Escherichia coli are the most commonly isolated microorganism in neonates with a deep neck abscess [12].

\section{Streptoccocal infections and PCV7 vaccination}

Streptococcus pneumoniae has been an important cause of a variety of head and neck infections. A recent retrospective review of the Kids National Inpatient Database found that there has been a decrease in reports of meningitis, mastoiditis, periorbital cellulitis, and Bezold abscess due to $S$. pneumoniae as a result of the introduction of the PCV7 vaccine [13]. However, despite the decreased incidence of these infections there has actually been an increase in admission from the emergency department for these diagnoses for children age 1-4 and an increase in the costs of providing care for these conditions. It is thought that this may be related to the selection of more pathogenic subtypes.

\section{Fusobacterial infections}

Fusobacterium necrophorum is a Gram-negative bacteria which is an obligate anaerobe. It is frequently found in peritonsillar abscesses and associated deep neck space infections which can lead to Lemierre syndrome (thrombophlebitis of the internal jugular vein (IJV).) A recent study examined the prevalence of F. necrophorum as a pathogen in acute tonsillitis and found that it was present in $16 \%$ of acute tonsillitis patients compared with $9 \%$ of control patients [14]. F. necrophorum was also reported recently as a cause of a retropharyngeal abscess in a patient following cervical trauma, the patient did not have associated Lemierre syndrome [15]. Infections with this organism are treated with culture directed antibiotics and surgery as indicated. Resistance to macrolides is common so when a Fusobacterial infection might be suspected, macrolides are poor empiric therapy choices.

\section{Initial antimicrobial treatment for the child with a neck infection}

Decisions regarding initial antimicrobial treatment in children should be made based on the incidence of various organisms and antimicrobial sensitivities in the child's geographic region. In California, where the authors practice, Table 1 shows our empiric antibiotic protocol. 


\section{Table 1. Empiric antibiotic protocol}

\author{
Infection \\ Peri-tonsillar, parapharyngeal or retropharyngeal \\ abscess submandibular space infection \\ Lemierre syndrome (submandibular thrombophlebitis \\ of IJV) \\ Cervical lymphadenitis (Beta hemolytic streptococci \\ or MSSA) \\ Cervical lymphadenitis (MRSA) \\ Non-tuberculous mycobacteria
}

\author{
Empiric antibiotic choice \\ Ampicillin/sulbactam or clindamycin; add vancomycin for toxic \\ appearing patients pending culture result \\ Ampicillin/sulbactam or ceftriaxone + metronidazole \\ Amoxicillin/clavulanate or cephalexin or clindamycin \\ Clindamycin or trimethroprim/sulfamethoxazole or vancomycin \\ Surgical therapy versus watchful waiting. Medical treatment \\ controversial
}

Incidence of deep neck abscesses and surgical intervention

\section{Incidence of deep neck space infections}

A study of the Kids' Inpatient Database was used to evaluate the incidence of retropharyngeal abscesses, peritonsillar abscesses, parapharyngeal abscesses and combined deep neck abscesses over the period from 2000 to 2009. It was found that there was a significant increase in the incidence of retropharyngeal abscesses in 2009 when compared with 2000 ( 0.22 cases per 10,000 in 2009 vs. 0.10 cases per 10,000 in $2000, p=0.02)$ [ $16 \bullet \bullet$. However, there was no significant change in the incidence of peritonsillar abscesses, parapharyngeal abscesses or combined deep neck abscesses.

\section{Rates of surgical intervention}

While the incidence of retropharyngeal abscesses was found to be increasing, the rate of surgical intervention for these cases decreased in 2009 (38\% of cases) relative to 2000 ( $48 \%$ of cases), $p=0.04$ [16••]. In cases of peritonsillar abscesses, there was an increase in the rate of incision and drainage $(26.4 \%$ in 2000 vs. $33.7 \%$ in $2009, p<0.001)$ with a decrease in the rate of tonsillectomy ( $13 \%$ in 2000 vs. $7.8 \%$ in $2009, p<0.001$ ) [17•]. The overall rate of surgical intervention for all deep neck space abscesses was not significantly changed between 2000 and 2009 (51 vs. $47 \%, p=0.19)[16 \bullet \bullet]$.

\section{Cost of treatment of deep neck space infections}

Interestingly, while there was no change in the rate of surgical management, there was a significant increase in inflation adjusted hospital charges associated with deep neck space infection cases between 2000 and 2009 from $\$ 9486$ to $\$ 16,348, p=0.005$. This is despite the fact that there was also no increase in length of stay (2.78 days in 2000 vs. 2.68 days in 2009, $p=0.12$ ) nor in the rates of CT scans performed (5.3 in 2000 vs. $4.1 \%$ in $2009, p=0.36$ ) [16••]. One possible explanation may be related to the cost of intravenous antibiotics. The Kids' 
Inpatient database does not record specifics regarding the type of intravenous antibiotics used or the duration of therapy. Further epidemiological resource utilization studies may be indicated to explore this.

\section{Head and neck manifestations of Kawasaki disease}

\section{Kawasaki disease}

Kawasaki disease (KD) is an idiopathic systemic inflammatory vasculitis of small and medium sized vessels. The disease most commonly affects children between 6 months and 5 years of age although atypical Kawasaki disease can affect children outside this age range. There is a male to female ratio $1.5-1$ and a higher incidence in persons of Asian ethnicity. The etiology of KD is unknown and there is no diagnostic test. It is critical that KD be diagnosed to prevent cardiac complications including perivasculitis of the coronary arteries causing aneurysm formation, obstruction thrombosis, and myocardial infarctions. There is a $2 \%$ mortality rate associated with the presence of these complications making KD the most common cause of pediatric acquired heart disease in North America, Europe, and Japan [18]. The diagnosis is made based on a constellation of clinical criteria (Table 2) [19, 20].

Lymphadenopathy occurs in 50-60\% of patients with Kawasaki disease and is typically firm and nonfluctuant. In $12 \%$ of patients the initial presenting symptom is cervical lymphadenopathy [21]. The lymphadenopathy is typically unilateral and does not respond to oral antibiotics given empirically. The diagnosis of KD must be considered in any young child with cervical lymphadenopathy, no lymph node abscess, who continues to have high fevers in spite of antibiotic therapy. KD can also present with retropharyngeal edema and cellulitis which can mimic a retropharyngeal abscess, however clinically it is associated with less dysphagia and neck pain compared with retropharyngeal abscess and there is less likely to be rim-enhancing lesion noted on CT scan in cases of Kawasaki associated retropharyngeal cellulitis/edema [22-24].

Laboratory tests may reveal elevated C-reactive protein, platelet count, eosinophils, and erythrocyte sedimentation rate. Treatment consists of aspirin and intravenous immunoglobulin (IVIG). The aspirin is useful for management of fever and arthritis but it does not decrease the incidence of coronary artery aneurysms. The IVIG, when given within

\section{Table 2. Kawasaki clinical criteria}

Fever lasting 5 days or more ( 4 days if treatment with IVIG eradicates fever) plus at least four of the following clinical signs not explained by another disease process

1. Bilateral conjunctival injection (80-90\%)

2. Changes in the oropharyngeal mucous membranes (including $>1$ of the following: injected or fissured lips, strawberry tongue, injected pharynx) 80-90\%

3. Changes in the peripheral extremities, including erythema or edema of the hands and feet (acute phase) or periungual desquamation (convalescent phase) $(80 \%)$

4. Polymorphous rash, primarily truncal; nonvesicular (90\%)

5. Cervical lymphadenophathy: anterior cervical lymph node at least $1.5 \mathrm{~cm}$ in diameter (50\%) 
the first 10 days of illness, has been shown to decrease the incidence of coronary aneurysms from 17 to $4 \%$ [21]. Cardiac evaluation including an echocardiogram and EKG should be performed as well as referral to a pediatric cardiologist. The cervical lymphadenopathy is typically selflimiting and does not require additional treatment.

\section{Conclusions}

\section{Compliance with Ethical Standards}

\section{Conflict of Interest}

Christian Hochstim and Anna Messner declare that they have no conflict of interest.

Human and Animal Rights and Informed Consent

This article does not contain any studies with human or animal subjects performed by any of the authors.

\section{References and Recommended Reading}

Papers of particular interest, published recently, have been highlighted as:

- Of importance

$\bullet \quad$ Of major importance

1. Delides A, Manoli E, Papadopoulos M, Nikolopoulos T. Ultrasound-guided transoral drainage of a paediatric parapharyngeal abscess. J Laryngol Otol. 2014;128:1120-2.

2. $\quad$ Scholtz J-E et al. Evaluation of image quality and dose reduction of $80 \mathrm{kVp}$ neck computed tomography in patients with suspected peritonsillar abscess. Clin Radiol. 2015;70:e67-73. 
Describes the diagnostic accuracy and improved clarity of low voltage CT scans in diagnosing peritonsillar abscess. This could potentially be easily adopted more broadly in clinical use and result in reduced radiation exposure.

3.- Collins B, Stoner J a, Digoy GP. Benefits of ultrasound vs. computed tomography in the diagnosis of pediatric lateral neck abscesses. Int J Pediatr Otorhinolaryngol. 2014;78:423-6.

Describes the diagnostic accuracy of ultrasound as an alternative to CT scanning in deep neck abscesses.

4. Saat R et al. MR imaging features of acute mastoiditis and their clinical relevance. AJNR Am J Neuroradiol. 2015;36:361-7.

5. Brenner DJ. Should we be concerned about the rapid increase in CT usage? Rev Environ Health 25:63-8.

6.• Pearce MS et al. Radiation exposure from CT scans in childhood and subsequent risk of leukaemia and brain tumours: a retrospective cohort study. Lancet (London, England). 2012;380:499-505.

Highlights the risks associated with CT scans due to radiation exposure

7. Wichmann JL et al. Low-tube-voltage $80-\mathrm{kVp}$ neck CT: evaluation of diagnostic accuracy and interobserver agreement. AJNR Am J Neuroradiol. 2014;35:2376-81.

8. Neff L, Newland JG, Sykes KJ, Selvarangan R, Wei JL. Microbiology and antimicrobial treatment of pediatric cervical lymphadenitis requiring surgical intervention. Int J Pediatr Otorhinolaryngol. 2013;77:817-20.

9. Walker PC, Karnell LH, Ziebold C, Kacmarynski DSF. Changing microbiology of pediatric neck abscesses in Iowa 2000-2010. Laryngoscope. 2013;123:249-52.

$10 . \bullet$ Worley ML et al. Suppurative cervical lymphadenitis in infancy: microbiology and sociology. Clin Pediatr (Phila). 2015;54:629-34.

Important review of current bacteriology of infant neck abscesses.

11.•• Cheng J, Elden L, Elden L. Children with deep space neck infections: our experience with 178 children. Otolaryngol Head Neck Surg. 2013;148:1037-42. Important review of current bacteriology of pediatric neck abscesses.

12. Mutlu M, Dereci S, Aslan Y. Deep neck abscess in neonatal period: case report and review of literature. Int J Pediatr Otorhinolaryngol. 2014;78:577-82.
13. Walls A, Pierce M, Krishnan N, Steehler M, Harley EH. Pediatric head and neck complications of Streptococcus pneumoniae before and after PCV7 vaccination. Otolaryngol Head Neck Surg. 2015;152:336-41.

14. Kjærulff AMG, Thomsen MK, Ovesen T, Klug TE. Clinical and biochemical characteristics of patients with Fusobacterium necrophorum-positive acute tonsillitis. Eur Arch Otorhinolaryngol. 2015;272:1457-63.

15. Cheng J, Kleinberger AJ, Sikora A. Fusobacterium necrophorum in a pediatric retropharyngeal abscess: a case report and review of the literature. Ear Nose Throat J. 2014;93:E22-4.

16.• Novis SJ, Pritchett CV, Thorne MC, Sun GH. Pediatric deep space neck infections in U.S. children, 2000-2009. Int J Pediatr Otorhinolaryngol. 2014;78:832-6.

Describes significant trends in the incidence and management of deep neck abscesses.

17. Qureshi H et al. Trends in the management of pediatric peritonsillar abscess infections in the U.S., 2000-2009. Int J Pediatr Otorhinolaryngol. 2015;79:527-31.

Describes the changes in management of peritonsillar abscesses towards increased incision and drainage and decreased tonsillectomy.

18. Singh S, Vignesh P, Burgner D. The epidemiology of Kawasaki disease: a global update. Arch Dis Child. 2015;1-5. doi:10.1136/archdischild-2014-307536.

19. Sundel RP. Kawasaki disease. Rheum Dis Clin N Am. 2015;41:63-73. viii.

20. Bayers S, Shulman ST, Paller AS. Kawasaki disease: part I. Diagnosis, clinical features, and pathogenesis. J Am Acad Dermatol. 2013;69.

21. Yoskovitch A, Tewfik TL, Duffy CM, Moroz B. Head and neck manifestations of Kawasaki disease. Int J Pediatr Otorhinolaryngol. 2000;52:123-9.

22. Nomura $\mathrm{O}$ et al. Comparison of patients with Kawasaki disease with retropharyngeal edema and patients with retropharyngeal abscess. Eur J Pediatr. 2014;173:381-6.

23. Tona R et al. Risk factors for retropharyngeal cellulitis in Kawasaki disease. Auris Nasus Larynx. 2014;41:455-8.

24. Puhakka L et al. Retropharyngeal involvement in Kawasaki disease-a report of four patients with retropharyngeal edema verified by magnetic resonance imaging. Int J Pediatr Otorhinolaryngol. 2014;78:1774-8. 